\title{
Extração de gelatina a partir das peles de cabeças de carpa comum
}

\author{
Gelatin extraction from skins of common carp heads
}

\section{Roberto de Souza Gomes da Silva ${ }^{\mathrm{I}}$ Sidney Fernandes Bandeira ${ }^{\mathrm{I}}$ Fabiane Cristina Petry ${ }^{\mathrm{I}}$ Luiz Antonio de Almeida Pinto ${ }^{*}$}

\section{RESUMO}

Neste estudo, foi avaliada a extração de gelatina a partir de cabeças de carpa comum (Cyprinus carpio) provenientes da industrialização. Para tanto, foram estudados os efeitos da concentração alcalina, o tempo de tratamento $e$ troca de solução alcalina na etapa de tratamento das cabeças de carpa. Foi utilizado um delineamento experimental em estrutura fatorial completa com três fatores em dois níveis de variação. Os fatores de estudo foram concentração de solução de $\mathrm{NaOH}$ (3-4mol $\left.L^{-1}\right)$, tempo de tratamento (45-105min) e troca de solução alcalina no tratamento, tendo como respostas rendimento em gelatina e força do gel. A condição mais adequada para se obter gelatina com bom rendimento (1,98\%) e força do gel $(240,3 \mathrm{~g})$ foi utilizando solução alcalina $3 \mathrm{~mol} \mathrm{~L}^{-1}$, tratamento de $105 \mathrm{~min}$ e com troca de solução de $\mathrm{NaOH}$.

Palavras-chave: aproveitamento de resíduos, gelatina, peles de pescado, rejeitos de pescado.

\section{ABSTRACT}

The aim of the present research was to study the gelatin extraction from skin of common carp heads (Cyprinus carpio). The effects of alkaline concentration, pre-treatment time and change of alkaline solution in pre-treatment step of carp heads were evaluated. It was used a complete experimental design with three factors and two variation levels. Pre-treatment time (45-105min), concentration of alkaline solution (3-4mol $\left.L^{-1}\right)$ and pre-treatment with change of alkaline solution were chosen as independent variable and gelatin yield and gel strength were the response variable. The best condition to obtain gelatin with good yield (1.98\%) and gel strength (240.3g) was using alkaline solution $3 \mathrm{~mol} \mathrm{~L}^{-1}$, pre-treatment time of $105 \mathrm{~min}$ and with one change of $\mathrm{NaOH}$ solution.

Key words: common carp, fish skins, fish waste, gelatin.

\section{INTRODUÇÃO}

No Brasil, muitas pisciculturas utilizam carpa comum. Diversas razões contribuem para que a carpa comum seja considerada um excelente peixe para pisciculturas e explicam a sua distribuição por todo o planeta: tolera baixos níveis de oxigênio dissolvido na água; reproduz-se facilmente em cativeiro; tolera as práticas de manejo para recria e propagação; apresenta grande tolerância a variações de temperatura $\left(\operatorname{de~} 4^{\circ} \mathrm{C}\right.$ até $35^{\circ} \mathrm{C}$ ); é espécie onívora que aceita e converte bem os mais variados tipos de alimentos de origem animal ou vegetal; adapta-se bem a sistemas de produção baseados na reciclagem de subprodutos agropecuários (ECHEVENGUÁet al., 2008).

Os rejeitos gerados durante o processamento da carpa (peles, cabeças, espinhaços, nadadeiras e vísceras) podem totalizar $60 \%$ da matériaprima, sendo que para a carpa comum, somente as cabeças representam aproximadamente $22 \%$ da matériaprima (KOLODZIEJSKA et al., 2008; ECHEVENGUÁ et al., 2008). Em busca de alternativas viáveis para aproveitar rejeitos de pescados, vários produtos podem ser obtidos, a exemplo da produção de hidrolisados protéicos e extração de colágeno e gelatina, aumentando o faturamento das empresas e reduzindo problema ambiental (BANDEIRA, 2009; KLOMKLAO et al., 2007). Existem poucos estudos relatados sobre a extração de gelatina a partir de cabeças de pescados.

'Universidade Federal do Rio Grande (FURG), Rua Engenheiro Alfredo Huch, 475, 96201-900, Rio Grande, RS, Brasil. E-mail: dqmpinto@furg.br. *Autor para correspondência. 
ARNESEN \& GILDBERG (2006) e LIU et al. (2008) estudaram o processo de extração da gelatina de cabeça de bacalhau e bagre, respectivamente. No entanto, nenhuma informação sobre gelatinas a partir de cabeça de carpa e suas propriedades tem sido relatado.

A gelatina é uma proteína derivada da hidrólise parcial do colágeno animal, contido em ossos e peles, principalmente de suínos e bovinos (YANG et al., 2007). A conversão do colágeno em gelatina pode ser obtida através do aquecimento do colágeno, em meio ácido ou alcalino. Gelatinas obtidas por tratamento ácido são designadas do tipo A, enquanto que as do tipo B são as obtidas por tratamento alcalino (WARDS \& COURTS, 1977). O processo produtivo de obtenção da gelatina consiste de três etapas: tratamento da matéria-prima, extração da gelatina e purificação/ secagem (KARIM \& BHAT, 2008).

As propriedades funcionais das gelatinas são dependentes das suas propriedades físico-químicas e estruturais, que serão determinantes para definir sua aplicabilidade (WARDS \& COURTS, 1977). A qualidade de uma gelatina é determinada por parâmetros como rigidez do gel, viscosidade, capacidade de intumescência (PARDI et al., 1996). A força do gel é a principal propriedade da gelatina e esta característica determina seu valor comercial (CHO et al., 2004).

Este trabalho teve por objetivo avaliar os efeitos da concentração alcalina, tempo de tratamento e troca de solução alcalina no processo de extração de gelatina das peles de cabeças de carpa comum (Cyprinus carpio).

\section{MATERIAL E MÉTODOS}

Matéria-prima

As cabeças de carpa foram obtidas de piscicultores da cidade de Roca Sales-RS descartadas na industrialização. $\mathrm{O}$ transporte do material coletado foi feito em caixas térmicas com gelo até o Laboratório de Operações Unitárias da Escola de Química e Alimentos (EQA), da Universidade Federal do Rio Grande (FURG). Posteriormente, foram acondicionadas em embalagens plásticas e freezer, armazenados a $-18^{\circ} \mathrm{C}$.

O preparo das amostras foi realizado segundo ARNESEN \& GILDBERG (2006). As cabeças de carpa comum foram moídas ( $5 \mathrm{~mm}$ ), lavadas com água $(1: 6 \mathrm{p} / \mathrm{v})$ a $4^{\circ} \mathrm{C}$ por $10 \mathrm{~min}$ e centrifugadas a $7000 \times g$ por 5 min para eliminação do excesso de água.

Tratamento das amostras e extração da gelatina

As cabeças moídas de carpa foram submetidas ao tratamento alcalino, com adição de água destilada (1:1 p/v) e ajuste de $\mathrm{pH}$ para 11, utilizando solução de $\mathrm{NaOH} 3$ e $4 \mathrm{~mol} \mathrm{~L}^{-1}$.

Nos processos sem troca de solução alcalina, a matéria-prima permaneceu em solução alcalina por um período de $45 \mathrm{~min}$ e $105 \mathrm{~min}$ (dependendo do experimento da matriz do planejamento apresentada na tabela 1), e o material tratado foi centrifugado a $4000 \times \mathrm{g}$ por $15 \mathrm{~min}$. Após, o precipitado foi suspenso em água destilada (1:1 p/v), ajustando-se o $\mathrm{pH}$ em 2, com solução de $\mathrm{HCl} 3 \mathrm{~mol} \mathrm{~L}^{-1}$, por $15 \mathrm{~min}$. O material então foi centrifugado a $4000 \times g$ por $15 \mathrm{~min}$.

Nos processos envolvendo troca de solução alcalina, a solução foi removida por centrifugação após $15 \mathrm{~min}$. Em seguida, foi feita a renovação da solução alcalina com adição de água destilada $(1: 1 \mathrm{p} / \mathrm{v})$, ajustando-se novamente o $\mathrm{pH}$ em 11 com solução de $\mathrm{NaOH} 3$ e $4 \mathrm{~mol} \mathrm{~L}^{-1}$. Nesse tratamento, o material permaneceu em solução nos tempos necessários para complementar os valores da matriz do planejamento experimental (Tabela 1) e, após esse período, foi centrifugado a $4000 \times \mathrm{g}$ por $15 \mathrm{~min}$. O tratamento com solução de $\mathrm{HCl} 3 \mathrm{~mol} \mathrm{~L}^{-1}$ deste processo seguiu-se como descrito no tratamento sem troca de solução alcalina.

Tabela 1 - Matriz do planejamento fatorial da etapa de tratamento das cabeças moídas de carpa nas formas codificadas e não-codificadas.

\begin{tabular}{lccccc}
\hline $\operatorname{Exp}\left(\mathrm{n}^{\mathrm{o}}\right)$ & $\mathrm{CA}\left(\mathrm{mol} \mathrm{L}^{-1}\right)$ & $\mathrm{t}(\mathrm{min})$ & $\mathrm{TS}$ & $\mathrm{RG}(\%)^{*}$ & $\mathrm{FG}(\mathrm{g})^{*}$ \\
\hline 1 & $3(-1)$ & $45(-1)$ & Sem (-1) & $2,27 \pm 0,04$ & $215,7 \pm 4,1$ \\
2 & $4(1)$ & $45(-1)$ & Sem $(-1)$ & $2,02 \pm 0,06$ & $268,6 \pm 6,2$ \\
3 & $3(-1)$ & $105(1)$ & Sem (-1) & $1,63 \pm 0,09$ & $298,7 \pm 6,8$ \\
4 & $4(1)$ & $105(1)$ & Sem (-1) & $1,49 \pm 0,08$ & $250,7 \pm 6,6$ \\
5 & $3(-1)$ & $45(-1)$ & Com (1) & $1,66 \pm 0,06$ & $248,9 \pm 3,8$ \\
6 & $4(1)$ & $45(-1)$ & Com (1) & $1,61 \pm 0,07$ & $277,4 \pm 6,3$ \\
7 & $3(-1)$ & $105(1)$ & Com (1) & $1,98 \pm 0,06$ & $240,3 \pm 6,3$ \\
8 & $4(1)$ & $105(1)$ & Com (1) & $1,69 \pm 0,08$ & $182,8 \pm 5,4$ \\
\hline
\end{tabular}

Exp=experimento; $\mathrm{CA}=$ concentração alcalina; $\mathrm{t}=$ tempo de tratamento da matéria prima; $\mathrm{TS}=$ troca de solução alcalina; $\mathrm{RG}=\mathrm{rendimento}$ em gelatina; FG=força do gel; *Média \pm erro padrão (para três repetições).

Ciência Rural, v.41, n.5, mai, 2011. 
O processo de extração da gelatina das amostras tratadas foi realizado com adição de água destilada $(1: 1 \mathrm{p} / \mathrm{v})$ a $52^{\circ} \mathrm{C}$, em banho termostatizado, por período de $2 \mathrm{~h}$ e $\mathrm{pH}$ ajustado em $4 \mathrm{com} \mathrm{HCl} 3 \mathrm{~mol} \mathrm{~L}^{-1}$. O recolhimento do sobrenadante (solução de gelatina) foi realizado após centrifugação a $4000 \times g$ por $15 \mathrm{~min}$. A solução de gelatina foi filtrada em funil de Büchner com papel filtro Whatman $\mathrm{n} .4$.

\section{Composição química das amostras}

As composições centesimais das cabeças moídas e das cabeças intumescidas foram determinadas em triplicata, segundo metodologias AOAC (1995). A umidade foi determinada pelo método gravimétrico (n.950.46) em estufa a $105^{\circ} \mathrm{C}$. O teor de proteína bruta foi determinado através do teor de $\mathrm{N}$-total pelo método de Kjeldahl (n.928.08), utilizando o fator de multiplicação de 6,25. O teor de lipídios, pelo método de Soxhlet ( $\left.n^{\circ} 960.39\right)$ e o teor de cinzas por método gravimétrico (n.920.153) em mufla a 500-600 ${ }^{\circ} \mathrm{C}$.

Caracterização das soluções de gelatina

Rendimento em gelatina

Para determinação do rendimento, foi utilizado o método descrito por YANG et al. (2007) com o rendimento calculado segundo a Equação 1.

$\mathrm{RG}=\frac{\mathrm{C}_{\mathrm{g}} \cdot \mathrm{V}_{\mathrm{s}}}{\mathrm{m}_{\mathrm{c}}}$

em que $\mathrm{RG}$ é o rendimento em gelatina ( $\mathrm{g}_{\text {gelatina }} / 100$ $\left.\mathrm{g}_{\text {cabeças }}\right), \mathrm{C}_{\mathrm{g}}$ a concentração de proteínas da solução de gelatina $\left(\mathrm{g} \mathrm{mL}^{-1}\right), \mathrm{V}_{\mathrm{s}} \mathrm{O}$ volume da solução de gelatina extraída $(\mathrm{mL})$ e $\mathrm{m}_{\mathrm{c}}$ a massa inicial da cabeça moída $(\mathrm{g})$. Força do gel

A força do gel foi determinada baseando-se na metodologia aplicada por YANG et al. (2007) e as determinações foram feitas com três repetições.

\section{Análise estatística}

Os fatores de estudo foram concentração alcalina $\left(\mathrm{X}_{1}\right)$, tempo de tratamento $\left(\mathrm{X}_{2}\right)$ e troca de solução de $\mathrm{NaOH}\left(\mathrm{X}_{3}\right)$, utilizados na etapa de tratamento das cabeças moídas de carpa. Os valores dos níveis estudados foram determinados a partir de ensaios preliminares e de valores apresentados na literatura (BANDEIRA, 2009; ARNESEN \& GILDBERG, 2006), e estão apresentados na tabela $1 \mathrm{em}$ suas formas codificadas e não-codificadas, para o nível de significância de $95 \%$.

Os experimentos foram realizados em réplicas e as respostas analisadas foram o rendimento em gelatina (RG) e força do gel (FG).
A análise estatística foi realizada com o auxílio do software Statistica ${ }^{\circledR} 6.0$ (Statsoft, USA), aplicando a metodologia do planejamento fatorial completo, utilizando os três fatores de estudo, com os seus dois valores. Os modelos estatísticos teóricos representados pelas equações obtidas para cada resposta foram na forma polinomial, considerando os efeitos principais dos fatores de estudo e seus efeitos de interação de primeira ordem, conforme apresentado na Equação 2 (BOX et al., 1978).

$\mathrm{Y}=\mathrm{A}+\mathrm{BX}_{1}+\mathrm{CX}_{2}+\mathrm{DX}_{3}+\mathrm{EX}_{1} \mathrm{X}_{2}+\mathrm{FX}_{1} \mathrm{X}_{3}+\mathrm{GX}_{2} \mathrm{X}_{3}+$ $\mathrm{HX}_{1} \mathrm{X}_{2} \mathrm{X}_{3}$ em que $Y$ é a resposta considerada no valor real, $\mathrm{X}_{1}, \mathrm{X}_{2}$ e $X_{3}$ são os fatores de estudo na forma codificada, $A$, B, C, D, E, F, G, H coeficientes de ajuste.

Através dos modelos estatísticos, foram construídos os cubos de respostas, utilizando o software Statistica ${ }^{\circledR}$ 6.0. Os cubos são as representações gráficas dos valores teóricos calculados pelos modelos, sendo estes posicionados nos vértices, e as arestas dos cubos representam os fatores de estudos com seus níveis na forma codificada (BOXet al., 1978).

\section{RESULTADOS E DISCUSSÃO}

Composição química das amostras

As cabeças moídas de carpa apresentaram $75,6 \pm 1,6 \%$ de umidade, proteínas $9,9 \pm 0,5 \%$, lipídios $3,8 \pm 0,6 \%$ e cinzas $9,1 \pm 0,8 \%$. A composição química para as cabeças intumescidas foi: umidade $57,7 \pm 1,4 \%$, proteínas $17,4 \pm 0,6 \%$, lipídios $1,7 \pm 0,2 \%$ e cinzas $22,1 \pm 1,2 \%$.

O teor de proteínas das cabeças intumescidas $(17,4 \%$ para uma massa em torno de $180 \mathrm{~g})$ representa a máxima produção possível de gelatina que pode ser extraída das cabeças. Mesmo com a redução da quantidade total de proteínas, as cabeças intumescidas apresentaram aumento percentual de proteínas em função da menor umidade do material, o que eleva o teor dos outros componentes.

Enquanto o teor de lipídios das cabeças intumescidas mostrou uma redução em relação ao teor inicial das éabeças moídas, em função da eficiência na remoção deste componente durante a etapa dos tratamentos, o teor de cinzas foi aumentado em função da redução de umidade no material.

Análise estatística dos resultados

Os valores de rendimento em gelatina das peles das cabeças (Tabela 1) permaneceram entre 1,50 a 2,3\% de gelatina. Segundo a literatura, o rendimento de extração de gelatina de pescado (6\%) é inferior ao 
da gelatina de mamíferos (19\%) (JAMILAH \& HARVINDER, 2002). De acordo com KARIM \& BHAT (2008), o processo de extração da gelatina depende dos parâmetros do processo (temperatura, tempo e $\mathrm{pH}$ ), do tratamento e da quantidade de colágeno na matériaprima.

A força do gel está na faixa de 50 a $300 \mathrm{~g}$ para gelatina de mamíferos, PARDI et al. (1996). No entanto, segundo KARIM \& BHAT (2008) para gelatina de pescado, o valor típico pode chegar a $270 \mathrm{~g}$. Os valores de força do gel (Tabela 1) ficaram dentro da faixa citada por KARIM \& BHAT (2008). A propriedade de força do gel é afetada por muitos fatores, tais como a massa molar, concentração da solução de gelatina, tempo e temperatura de maturação do gel e pH (KASANKALA et al., 2007).

Pode-se observar, na tabela 2, referente à análise de variância (ANOVA) das respostas consideradas, que, para o rendimento em gelatina, todos os efeitos principais e a interação entre o tempo de tratamento e o tratamento com ou sem troca de solução de $\mathrm{NaOH}\left(\mathrm{X}_{2} \mathrm{X}_{3}\right)$ foram significativos ao nível de $95 \%(\mathrm{P} \leq 0,05)$. Para a força do gel, considerando o mesmo nível de significância de $95 \%(\mathrm{P} \leq 0,05)$, apenas as variáveis concentração alcalina $\left(X_{1}\right)$ e a interação desta variável com o tratamento com ou sem troca de
$\mathrm{NaOH}\left(\mathrm{X}_{1} \mathrm{X}_{3}\right)$ não produziram um efeito significativo na resposta.

Os modelos teórico-estatísticos representados pelas equações 3 e 4 foram obtidos a partir das análises de regressão para as respostas rendimento em gelatina $\left(\mathrm{Y}_{\mathrm{RG}}\right)$ e força do gel $\left(\mathrm{Y}_{\mathrm{FG}}\right)$, considerando apenas os efeitos principais e suas interações na forma codificada, os quais foram significativos na análise ao nível de $95 \%(P \leq 0,05)$. $\mathrm{Y}_{\mathrm{RG}}=1,79-0,18 \mathrm{X}_{1}-0,19 \mathrm{X}_{2}-0,12 \mathrm{X}_{3}+0,39 \mathrm{X}_{2} \mathrm{X}_{3}$ (3)

$\mathrm{Y}_{\mathrm{FG}}=247,9-4,76 \mathrm{X}_{2}-10,53 \mathrm{X}_{3}-23,37 \mathrm{X}_{1} \mathrm{X}_{2}-21,03 \mathrm{X}_{2} \mathrm{X}_{3}$ (4)

Rendimento em gelatina e força do gel

Os cubos de resposta para o rendimento em gelatina e força do gel estão representados pela figuras 1a e $1 \mathrm{~b}$, respectivamente, das gelatinas obtidas da extração das peles das cabeças de carpa. Os valores nos vértices representam os valores teóricos calculados pelo modelo estatístico representado pelas Equações 3 e 4 e, nas arestas, estão representados os níveis codificados dos fatores de estudo.

A figura la mostra que o maior rendimento em gelatina foi obtido com tratamento sem troca de solução de $\mathrm{NaOH}(-1)$, em menor tempo de tratamento

Tabela 2 - ANOVA para a resposta rendimento e força do gel das gelatinas obtidas da extração das peles de cabeças de carpa.

\begin{tabular}{|c|c|c|c|c|c|}
\hline \multicolumn{6}{|c|}{ Rendimento em gelatina } \\
\hline Variável & SQ & GL & QM & $\mathrm{F}$ & $P$ \\
\hline $\mathrm{X}_{1}$ & 0,133225 & 1 & 0,133225 & 13,950 & 0,0057 \\
\hline $\mathrm{X}_{2}$ & 0,148225 & 1 & 0,148225 & 15,521 & 0,0043 \\
\hline $\mathrm{X}_{3}$ & 0,055225 & 1 & 0,055225 & 5,783 & 0,0428 \\
\hline $\mathrm{X}_{1} \mathrm{X}_{2}$ & 0,004225 & 1 & 0,004225 & 0,442 & 0,5246 \\
\hline $\mathrm{X}_{1} \mathrm{X}_{3}$ & 0,000625 & 1 & 0,000625 & 0,065 & 0,8045 \\
\hline $\mathrm{X}_{2} \mathrm{X}_{3}$ & 0,616225 & 1 & 0,616225 & 64,526 & $<0,0001$ \\
\hline Falta de ajuste & 0,030625 & 1 & 0,030625 & 3,207 & 0,1111 \\
\hline Erro puro & 0,076400 & 8 & 0,009550 & & \\
\hline Total & 1,064775 & 15 & & & $\mathrm{R}^{2}=89,9 \%$ \\
\hline Variável & SQ & GL & QM & $\mathrm{F}$ & $P$ \\
\hline $\mathrm{X}_{1}$ & 145,81 & 1 & 145,806 & 2,202 & 0,1761 \\
\hline $\mathrm{X}_{2}$ & 361,95 & 1 & 361,951 & 5,467 & 0,0475 \\
\hline $\mathrm{X}_{3}$ & 1774,52 & 1 & 1774,516 & 26,805 & 0,0008 \\
\hline $\mathrm{X}_{1} \mathrm{X}_{2}$ & 8737,58 & 1 & 8737,576 & 131,986 & $<0,0001$ \\
\hline $\mathrm{X}_{1} \mathrm{X}_{3}$ & 288,15 & 1 & 288,151 & 4,353 & 0,0704 \\
\hline $\mathrm{X}_{2} \mathrm{X}_{3}$ & 7077,02 & 1 & 7077,016 & 106,903 & $<0,0001$ \\
\hline Falta de ajuste & 55,13 & 1 & 55,131 & 0,833 & 0,3881 \\
\hline Erro puro & 529,61 & 8 & 66,201 & & \\
\hline Total & 18969,75 & 15 & & & $\mathrm{R}^{2}=96,9 \%$ \\
\hline
\end{tabular}

SQ=soma quadrática; GL=graus de liberdade; $Q M=$ =quadrado médio; F=valor da estatística do teste; P=significância.

Ciência Rural, v.41, n.5, mai, 2011. 


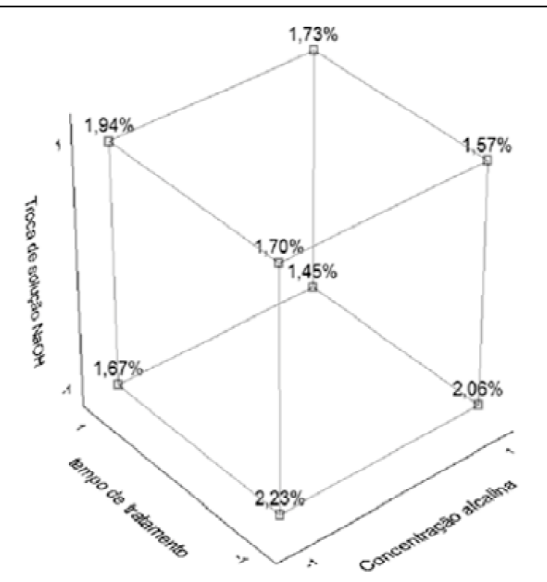

(a)

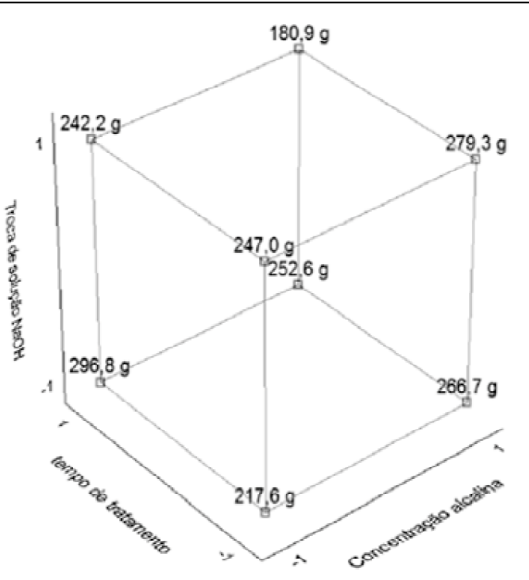

(b)

Figura 1 - Cubos de resposta para (a) rendimento em gelatina e (b) força do gel, obtidos da extração de gelatina das peles de cabeças de carpa.

(-1) e menor concentração alcalina (-1). O uso de menores concentrações de solução alcalina no tratamento das cabeças provocou aumento do volume final do material tratado e, por isso, pode ter favorecido maior dissolução do colágeno na forma de gelatina. Isso pode explicar o comportamento dos menores rendimentos nas condições aplicadas para maiores tempos de tratamento, já que, em tempos prolongados, a hidrólise das moléculas de colágeno é maior, sendo estas descartadas junto com o sobrenadante do tratamento após a centrifugação. De acordo com COLE (2010), as peles de pescados não possuem tantas ligações químicas, não havendo necessidade de um tratamento alcalino intenso e longo.

Analisando a figura $1 \mathrm{~b}$, é possível estabelecer a melhor faixa de trabalho quando se deseja extrair gelatinas mais rígidas. Os resultados mostram que a melhor condição se dá ao se trabalhar com maior tempo de tratamento $(+1)$, menor concentração alcalina (-1) e tratamento sem realização de troca de solução de $\mathrm{NaOH}(-1)$. Na condição mencionada, foi possível obter força do gel na faixa de $300 \mathrm{~g}$. No entanto, nessa condição de tratamento, o rendimento é baixo. $\mathrm{O}$ valor para a força do gel foi superior aos encontrados por YANG et al. (2007) e ALFARO (2008), que obtiveram valores de 225 e $221 \mathrm{~g}$ em gelatinas extraídas de peles de bagre e peles de tilápia, respectivamente. As características de temperatura da gelatina e do colágeno de peles de pescado refletem a temperatura do habitat natural do peixe. As gelatinas de peixes de água fria possuem menor conteúdo de aminoácidos (prolina e hidroxiprolina) que as gelatinas de peixes tropicais, e, portanto, apresentam melhores propriedades reológicas (BUENO, 2008).
Através das figuras 1a e 1b, pôde-se constatar que, para obtenção de gelatina que apresente bom rendimento e boa propriedade física, a condição mais adequada foi quando se utilizou menor concentração alcalina (-1), maior tempo de tratamento $(+1)$ e troca de solução de $\mathrm{NaOH}$ no tratamento (+1), que corresponde ao experimento 7 da tabela 1 .

\section{CONCLUSÃO}

Para o rendimento em gelatina, todos os efeitos principais e a interação entre o tempo e o tratamento com troca de solução alcalina foram significativos ao nível de $95 \%(\mathrm{P} \leq 0,05)$. Para a força do gel, considerando o mesmo nível de significância, apenas as variáveis concentração alcalina e a interação desta variável com o tratamento com troca de solução alcalina não produziram um efeito significativo na resposta $(P>0,05)$.

A condição mais adequada para extração de gelatina das peles de cabeças de carpa comum foi obtida na concentração de solução alcalina $3 \mathrm{~mol} \mathrm{~L}^{-1}$, tempo de tratamento de 105 min e com troca de solução alcalina. Nessa condição, os valores encontrados foram rendimento de $1,98 \%$ e força do gel de 240,3.

\section{REFERÊNCIAS}

ALFARO, A.T. Otimização das condições de extração e caracterização da gelatina de pele de tilápia (Oreochromis urolepis hornorum). 2008. 130f. Tese (Doutorado em Ciência e Tecnologia de Alimentos) - Universidade Federal de Pelotas, RS. 
AOAC. Association of Official Analytical Chemists. Official Methods of Analysis of AOAC international. 16.ed. Arlington, 1995. 1025p.

ARNESEN, J.A.; GILDBERG, A. Extraction of muscle proteins and gelatine from cod head. Process Biochemistry, v.41, n.3, p.697-700, 2006. Disponível em: <http://dx.doi.org/ 10.1016/j.procbio.2005.09.001>. Acesso em: 14 dez. 2009. doi: 10.1016/j.procbio.2005.09.001

BANDEIRA, S.F. Extração e caracterização da gelatina obtida de cabeças de carpa (Aristichthys mobilis). 2009. 72f. Dissertação (Mestrado em Engenharia e Ciência de Alimentos) - Universidade Federal do Rio Grande, RS

BOX, G.E.P. et al. Statistics for experimenters: an introduction to design, data analysis, and model building. New York : Wiley \& Sons, 1978. 530p.

BUENO, C.M.M. Extração e caracterização de gelatina de pele de tilápia e aplicação como agente encapsulante de óleo de salmão em micropartículas obtidas por coacervação complexa. 2008. 133f. Dissertação (Mestrado em Alimentos e Nutrição) - Universidade Estadual de Campinas, SP.

CHO, S.M. et al. Processing optimization and functional properties of gelatin from shark (Isurus oxyrinchus) cartilage. Food Hydrocolloids, v.18, n.4, p.575-579, 2004. Disponível em: <http://dx.doi.org/10.1016/j.foodhyd..2003.10.001>. Acesso em: 06 jan. 2010. doi: 10.1016/j.procbio.2005.09.001.

COLE, C.G.B. Gelatin food science. Disponível em: <http:/ /www.gelatin.co.za>. Online. Acesso em: 18 jan. 2010.

ECHEVENGUÁ, M.M. et al. Qualidade da polpa da carpa Húngara transportada viva ou no gelo. Ciência Rural, v.38, n.7, p.2004-2010, 2008. Disponível em: <http://www.scielo.br/ scielo.php ? script =sci_arttext \& pid=S010384782008000700032>. Acesso em: 08 jan. 2010. doi: 10.1590/ S0103-84782008000700032.

JAMILAH, B.; HARVINDER, K.G. Properties of gelatins from of fish - Black tilapia (Oreochromis mossambicus) and red tilapia (Oreochromis nilotica). Food Chemistry, v.77, n.1, p.81-84, 2002. Disponível em: <http://dx.doi.org/10.1016/ S0308-8146(01)00328-4>. Acesso em: 12 out. 2009. doi: 10.1016/S0308-8146(01)00328-4.

KARIM, A.A.; BHAT, R. Fish gelatin: properties, challenges, and prospects as an alternative to mammalian gelatins. Food Hydrocolloids, v.23, n. 3, p.563-576, 2008. Disponível em: <http://dx.doi.org/10.1016/j.foodhyd.2008.07.002>. Acesso em: 07 out. 2009. doi: 10.1016/j.foodhyd.2008.07.002.

KASANKALA, L.M. et al. Optimization of gelatine extraction from grass carp (Catenopharyngodon idella) fish by response surface methodology. Bioresource Technology, v.98, n.17, p.3338-3343, 2007. Disponível em: <http://dx.doi.org/ 10.1016/j.biortech.2006.03.019>. Acesso em: 07 out. 2009. doi: 10.1016/j.biortech.2006.03.019.

KLOMKLAO, S. et al. Purification and characterization of trypsins from the spleen of skipjack tuna (Katsuwonus pelamis). Food Chemistry, v.100, n.4, p.1580-1589, 2007. Disponível em: <http://dx.doi.org/10.1016/j.foodchem.2006.01.001>. Acesso em: 07 out. 2009. doi: 10.1016/ j.foodchem.2006.01.001.

KOLODZIEJSKA, I. et al. Effect of extracting time and temperature on yield of gelatin from different fish offal. Food Chemistry, v.107, n.2, p.700-706, 2008. Disponível em: <http://dx.doi.org/10.1016/j.foodchem.2007.08.071>. Acesso em: 07 out. 2009. doi: 10.1016/ j.foodchem.2007.08.071.

PARDI, M.C. et al. Ciência, higiene e tecnologia da carne. Goiânia: CEGRAF/EDUF, 1996. V2.

YANG, H. et al. 2-Step optimization of the extraction and subsequent physical properties of channel catfish (Ictalurus punctatus) skin gelatin. Food Chemistry and Toxicology, v.72, n.4, p.188-195, 2007. Disponível em: <http://dx.doi.org/ 10.1111/j.1750-3841.2007.00319.x>. Acesso em: 21 nov. 2009. doi: 10.1111/j.1750-3841.2007.00319.x.

WARDS, A.G.; COURTS, A. The science and technology of gelatin. New York: Academic, 1977. 580p. 